\title{
Iron Chelation with Transdermal Deferoxamine Accelerates Healing of Murine Sickle Cell Ulcers
}

\author{
Melanie Rodrigues, ${ }^{1}$ Clark A. Bonham, ${ }^{1}$ Caterina P. Minniti, \\ Kalpna Gupta, Michael T. Longaker, and Geoffrey C. Gurtner ${ }^{1, *}$ \\ ${ }^{1}$ Department of Surgery, Stanford University School of Medicine, Stanford, California. \\ ${ }^{2}$ Division of Hematology, Montefiore Medical Center, Albert Einstein College of Medicine, Bronx, New York. \\ ${ }^{3}$ Division of Hematology, Oncology, and Transplantation, Department of Medicine, University of Minnesota, \\ Minneapolis, Minnesota.
}

Objective: Sickle cell ulcers (SCUs) are a devastating comorbidity affecting patients with sickle cell disease (SCD). SCUs form over the medial or lateral malleoli of the lower extremity, are slow to heal, and prone to recidivism. Some SCUs may never heal, leading to chronic pain and foot deformities. There is no specific and effective therapy for SCUs. Systemic deferoxamine (DFO) has been demonstrated to prevent some of the sequelae of SCD by chelating iron. In this study, we tested the ability of DFO delivered via a transdermal delivery system (DFO-TDDS) to accelerate healing in a murine model of SCU.

Approach: Excisional wounds were created in a transgenic murine model of SCD expressing $>99 \%$ human sickle hemoglobin, and healing rates were compared with wounds in wild-type mice. Next, excisional wounds in SCD mice were treated with DFO-TDDS, DFO injection, or left untreated. Wound closure rates, histology, and iron in the healed wounds were analyzed.

Results: Wounds in SCD mice healed significantly slower than wild-type mice $(* * * p<0.001)$. DFO-TDDS-treated wounds demonstrated significantly accelerated time to closure, reduced size, and improved wound remodeling compared with untreated wounds $\left({ }^{* * *} p<0.001\right)$ and DFO injection treatment $(* p<0.05)$. DFO released from the TDDS into wounds resulted in chelation of excessive dermal-free iron.

Innovation: DFO-TDDS is a novel therapeutic that is effective in healing wounds in sickle cell mice.

Conclusion: DFO-TDDS significantly accelerates healing of murine SCUs by chelation of excessive free iron and is currently manufactured in an FDAcompliant facility to be translated for treating human SCUs.

Keywords: sickle cell disease, sickle cell ulcers, deferoxamine, wound healing, iron chelator

\section{INTRODUCTION}

The US Center for Disease Control estimates that $\sim 100,000$ people in the United States live with sickle cell disease (SCD). ${ }^{1}$ Approximately $2.5 \%$ of SCD patients experience sickle cell ulcers (SCUs), with the incidence increasing to $27 \%$ in patients living in low-income areas. ${ }^{2}$ SCD results from a mutation of the hemoglobin gene that generates abnormal hemoglobin (Hemoglobin S), which polymerizes when deoxygenated and damages the erythrocyte membrane causing sickle-shaped erythrocytes. ${ }^{3,4}$ Abnormal rheology and increased hemolysis make sickle cell patients anemic and cause abnormal microcirculation with

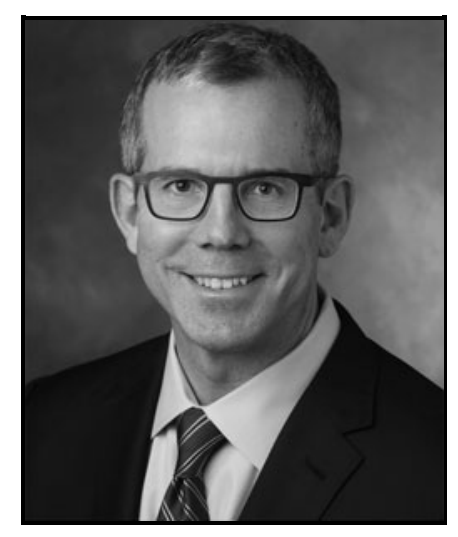

Geoffrey C. Gurtner, MD, FACS

Submitted for publication February 28, 2018 Accepted in revised form March 31, 2018.

*Correspondence: Department of Surgery, Stanford University School of Medicine, 257 Campus Drive West, GK-201, Stanford, CA, 94305 (e-mail: ggurtner@stanford.edu). 
repetitive episodes of hypoxia reperfusion injury and local ischemic events that can lead to the formation of SCUs. ${ }^{5}$

Sickle cell erythrocytes also display an abnormal level of membrane-associated iron and excessive superoxide production. ${ }^{6}$ Furthermore, chronic hemolysis leads to an accumulation of heme in plasma and in tissues, ${ }^{7}$ resulting in increased reactive oxygen species (ROS) both through the Fenton reaction $^{8}$ and from neutrophil- and monocyte-derived oxidative burst. ${ }^{7}$ Increased ROS lead to tissue-free radical defense mechanisms, yielding DNA damage, excessive inflammation, and end-organ damage, among them, chronic ulceration. ${ }^{9,10}$ Acute vasoocclusive episodes, typical of SCD, result in severe pain, while ulcers are associated with both acute and chronic pain. ${ }^{11}$

Patients with SCD manifest the first SCUs in the second decade of life, ${ }^{12}$ but the prevalence increases with age. The majority of SCUs are slow to heal and form over the medial or lateral malleoli, but occasionally, the dorsum of the foot and the digits may be affected. ${ }^{13}$ With or without specialized care, these wounds can increase in size, become infected and reoccur. ${ }^{1}$ In some patients, ulcers can last indefinitely, leading to extreme pain, depression, and long-term deformities. ${ }^{14}$ Unfortunately, there is no specific and uniformly effective therapy for treating SCUs. ${ }^{13}$ Ideally, a vulnerary agent for SCU would chelate iron in the skin preventing the cascade leading to tissue necrosis.

Systemically delivered deferoxamine (DFO) has been FDA approved since 1968 for treating hemochromatosis in sickle cell patients. ${ }^{15}$ SCD patients receive repeated blood transfusions to increase normal hemoglobin and dilute hemoglobin S, which can lead to free iron accumulation and hemochromatosis, most critically in the liver and occasionally in the heart. ${ }^{10,16}$ To chelate excessive iron from their blood, SCD patients receive DFO, usually in the form of Desferal (Novartis, Switzerland). ${ }^{17}$ DFO maintains a maximal affinity for ferric iron, forming an exceptionally stable hexadentate ligand, ferrioxamine, following chelation. ${ }^{18,19}$ The drug is either delivered subcutaneously over $8-24 \mathrm{~h}$ with the use of a portable infusion pump (not to exceed $20-40 \mathrm{mg} / \mathrm{kg} /$ day), intravenously over $8-12 \mathrm{~h}(20-40 \mathrm{mg} / \mathrm{kg} / \mathrm{day}$ for children and $40-50 \mathrm{mg} / \mathrm{kg} /$ day for adults,) or intramuscularly, not exceeding a daily dose of $1,000 \mathrm{mg} .{ }^{17}$ There has been no evidence of toxicity in adult or pediatric patients when treated within these dose limits, ${ }^{20}$ but ototoxicity and retinal abnormalities are reported in higher doses (above $50 \mathrm{mg} / \mathrm{kg} /$ day).
We have previously demonstrated that DFO is an effective treatment for healing diabetic murine wounds. ${ }^{21,22}$ However, the major challenge has been effective topical delivery of this drug into the ulcer and intact skin for prophylactic use. DFO is a hydrophilic drug with a short half-life and delivery into the hydrophobic stratum corneum by injection or direct application in either liquid or cream form has been inconsistent and not always successful. Hence, we developed a novel transdermal delivery system for deferoxamine (DFO-TDDS) that uses reverse micelles to release DFO in a sustained manner to the healing dermis. ${ }^{23}$ We first applied DFO-TDDS on diabetic ulcers created in murine models. DFO-TDDS was effective in both preventing and accelerating wound healing in diabetic mice. $^{23}$

In this study, we hypothesize that DFO-TDDS can enhance healing of wounds in sickle cell mice through iron chelation. We first created excisional wounds in a transgenic sickle cell mouse model expressing $>99 \%$ human sickle hemoglobin ( $\mathrm{HbSS}$ BERK) and demonstrated that wound healing is impaired in these mice. Next, we treated excisional wounds in HbSS-BERK with DFO-TDDS or subcutaneously injected DFO. We discovered that DFOTDDS significantly accelerated healing of wounds in the HbSS-BERK mice by chelation of excessive free iron. Our findings demonstrate an effective therapeutic solution that can be rapidly translated into patients with SCUs.

\section{CLINICAL PROBLEM ADDRESSED}

There is currently no effective therapy for healing SCUs. Wound care for SCUs is labor intensive and requires multiple clinic visits on a weekly basis. Current treatment protocols include surgical debridement, which is performed to remove dead and infected tissue, and to allow granulation to occur. Compression and dressings that absorb excess exudate while maintaining a moist wound surface are also used. Despite these efforts, SCUs are slow to heal, if at all, and are prone to recurrence. ${ }^{24}$ Antibiotics are also prescribed for wounds with obvious purulence, cellulitis, or osteomyelitis, yet there are insufficient data to support either systemic or local antibiotic therapy for SCUs. In this study, we show that clinical-grade DFO-TDDS significantly accelerates healing of excisional wounds in sickle cell mice. Since DFO is approved for systemic use in patients with SCD, we anticipate that local DFOTDDS can be translated effectively to treat patients with SCUs. 


\section{MATERIALS AND METHODS Mice}

$H b S S$-BERK mice do not express mouse $\alpha$ - and $\beta$-globins but carry the transgenes for human $\alpha$ - and $\beta$-sickle genes on a mixed genetic background. ${ }^{25}$ These mice simulate human SCD, including hemolysis, reticulocytosis, anemia, extensive organ damage, shortened life span, and pain. ${ }^{25-27}$ Homozygous HbSS-BERK mice were bred and phenotyped for human sickle hemoglobin in Dr. Kalpna Gupta's laboratory at the University of Minnesota as previously described ${ }^{27}$ and transported to Stanford under a material transfer agreement. Genotyping for the knockout and hemoglobin transgenes was done by Transnetyx (Cordova, TN). Control mice (wild type) on a C57Bl/ 6 background [\#000664] were obtained from Jackson Laboratories (Bar Harbor, ME). All mice used in the experiments were housed in the Stanford University Veterinary Service Center. NIH and Stanford University animal care guidelines were followed. All procedures were approved by the university's Administrative Panel on Laboratory Animal Care.

\section{DFO-TDDS production}

Clinical-grade DFO-TDDS was manufactured, sterilized, and packaged in an FDA- and ISO-13485compliant facility (TauTona Group, Redwood City, CA) using a formulation previously described. ${ }^{23}$

\section{Excisional wound healing}

Five-month-old male $H b S S$-BERK and wild-type mice were subjected to a quantitative and reproducible model of excisional wounding using an established protocol. ${ }^{28}$ Briefly, after induction of anesthesia and removal of hair using a shaver and depilatory cream, two $6 \mathrm{~mm}$ full-thickness cutaneous wounds were excised on either side of the midline of the murine dorsum using a biopsy punch (Integra, NJ). Each wound was stented with silicone rings (Grace Biolabs, OR) with outer and inner diameters of 16 and $10 \mathrm{~mm}$, and sutured in place to prevent wound contraction. All mice were given buprenorphine slow-release at the time of wounding as analgesic.

\section{Wound treatment}

$H b S S$-BERK mice were randomized into three treatment groups: DFO-TDDS, DFO injection, or untreated. Following wounding, a $6 \mathrm{~mm}$ punch of DFO-TDDS was placed on the wound daily or $20 \mu \mathrm{L}$ of $100 \mathrm{mM}$ DFO solution was subcutaneously injected into the wound daily. The untreated group received no injection or patch. All wounds were covered with an occlusive dressing (Tegaderm, 3M;
St Paul, MN). Digital photographs were taken every other day. Wound area was measured using ImageJ software (NIH).

\section{Histology}

On closure, wounds were collected, fixed in $4 \%$ paraformaldehyde overnight, and embedded in paraffin. For analysis of dermal thickness, paraffin sections were stained with trichrome (SigmaAldrich) and average thickness was calculated from three measurements per high-power field per wound.

\section{Perl's Prussian blue stain}

Abcam iron stain kit (ab150674; Cambridge, UK) was used to display iron present in tissue sections. Histological sections were deparaffinized and rehydrated. Equal volumes of potassium ferrocyanide and hydrochloric acid solution (2\%) were combined to make the iron stain solution. Slides were incubated in the solution for $3 \mathrm{~min}$ and then rinsed with distilled water. Slides were then stained with Abcam nuclear fast red solution for $5 \mathrm{~min}$ and washed four separate times with distilled water. Slides were finally dehydrated in $95 \%$ ethanol, followed by absolute ethanol. Blue stain directly correlates with nonchelated iron in the skin. DFO chelates iron, forming ferrioxamine, which does not react in the Perl's Prussian blue reaction.

\section{Statistical analysis}

Results are presented as mean \pm SEM. Standard data analysis was performed using a Student's $t$-test. ANOVA was used to measure significance of wound closure. Results were considered significant for $* p \leq 0.05, * * p<0.01$, and $* * * p<0.001$.

\section{RESULTS}

\section{HbSS-BERK mice undergo wound healing impairments compared with wild-type mice}

$H b S S$-BERK and wild-type mice were splinted following wounding to minimize contracture and to replicate human-like wound healing kinetics. ${ }^{28}$ Images of the excisional wounds were taken every other day and the wound healing outcomes were assessed by comparing wound sizes at each time point to day 0 within each mouse (Fig. 1A). HbSSBERK mice demonstrated markedly delayed wound healing compared with wild-type control mice. Differences in the wound area were statistically significant at all time points from day 6 onward until closure $(* p<0.05, * * p<0.01, * * * p<0.001)$ (Fig. 1B). Time to complete wound closure in the $H b S S$-BERK mice and wild-type mice was $17.1429 \pm 0.4041$ and $13.4 \pm 0.3055$, respectively (Fig. 1C). These results indicate that the HbSS-BERK mice exhibit delayed wound healing. 

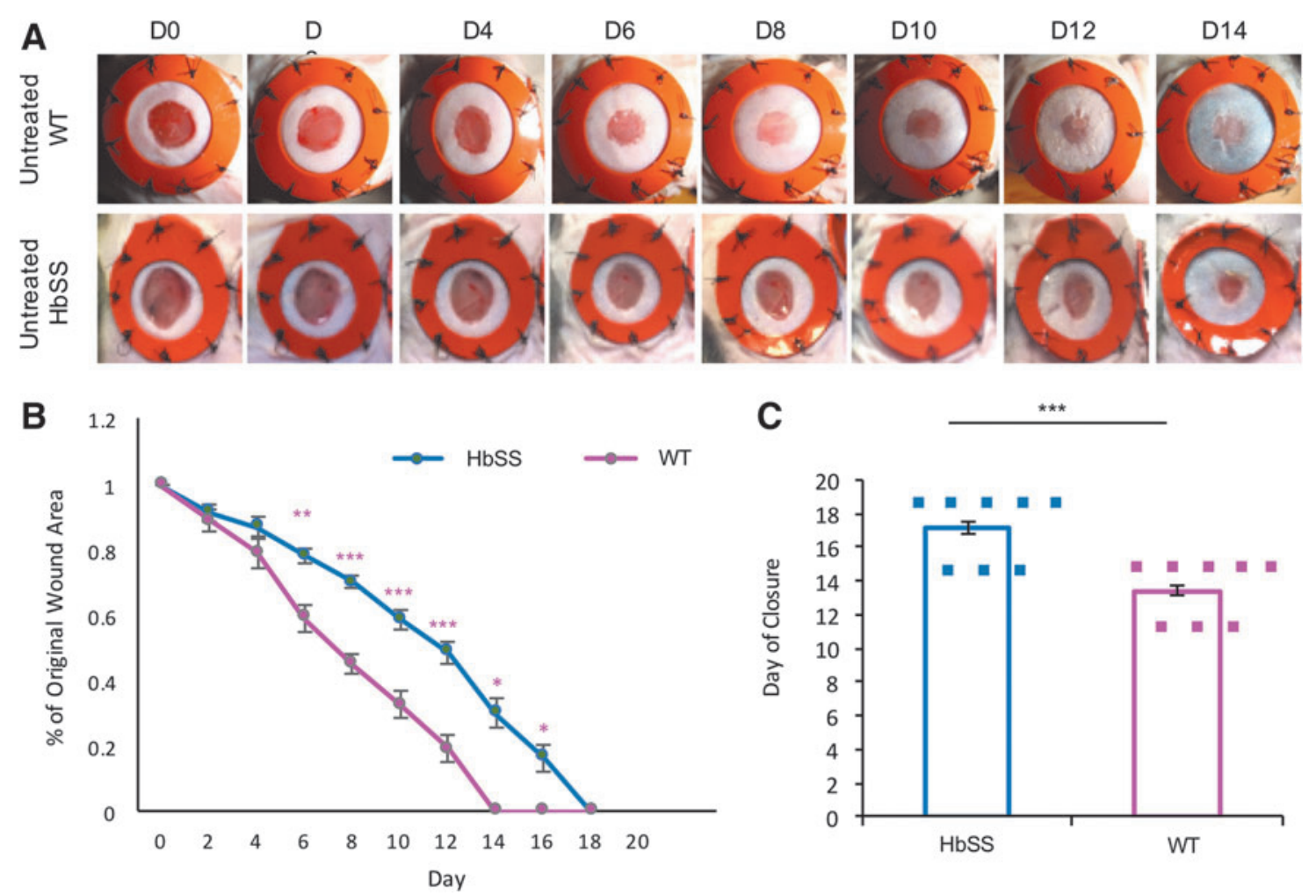

Figure 1. HbSS-BERK mice demonstrate wound healing impairments compared with wild-type mice. (A) Photographs of wounds taken every other day after wounding until closure in the untreated HbSS and wild-type groups. (B) Wound healing curves demonstrate reduction in wound size every other day until closure. (C) Denotes the time of closure of wounds. At least eight wounds were analyzed in each cohort. ${ }^{*} p<0.05,{ }^{* *} p<0.01,{ }^{* * *} p<0.001$ by ANOVA.

\section{HbSS-BERK wounds treated with DFO-TDDS demonstrate accelerated wound healing}

Once we established that $H b S S$-BERK mice have impaired wound healing, we treated these mice with DFO-TDDS or injected the wounds subcutaneously with DFO solution daily. Wound healing in the treated mice was compared with wounds in $H b S S$ BERK mice that were left untreated. DFO-TDDStreated mice displayed significantly accelerated wound closure compared with both the DFO injection $(* p<0.05)$ and the untreated group $(* * * p<0.001)$ (Fig. 2A, B). Injection with DFO solution significantly reduced wound area compared with untreated controls $(* * p<0.01)$, but not as effectively as the DFO-TDDS group. Time to complete wound closure in the DFO-TDDS group, DFO injection group, and untreated groups was $13 \pm 0.3660$, $14.5 \pm 0.6268$, and $17.1429 \pm 0.4041$, respectively. These results demonstrate that DFO-TDDS significantly accelerates wound healing and is more effective than DFO injection at treating wounds in a murine model of sickle cell ulceration.

\section{HbSS-BERK wounds treated with DFO-TDDS demonstrate a thicker dermis}

Histological sections of the healed wound were subjected to trichrome analysis to determine col- lagen deposition in the dermis. DFO-TDDS-treated wounds in the HbSS-BERK mice displayed markedly greater collagen deposition in organized bundles compared with the untreated group and the DFO injection-treated mice (Fig. 3A). The width of collagen across the slides was measured to determine thickness of the dermis. Wounds treated with DFO-TDDS demonstrated significantly higher dermal thickness $(* p<0.001)$ (Fig. 3B). Greater collagen deposition and a higher dermal thickness are desirable in the healed skin of sickle cell patients, to prevent a wound recurrence at the same site.

\section{DFO-TDDS accelerates wound healing in HbSS-BERK mice by chelation of free iron}

Since SCD is characterized by excessive free iron leading to tissue dysfunction, histological sections of the healed wound were subjected to Perl's Prussian blue stain to determine the presence of iron in untreated wounds and wounds treated with DFO. Excessive deposition of iron was observed in the untreated group, and these wound regions highly correlated with lesser dermal thickness and reduced dermal integrity (Fig. 4A). Both DFOTDDS and DFO injection decreased iron in the skin as evidenced by negligible levels of Perl's Prussian blue stain (Fig. 4A, B). However, the DFO-TDDS 


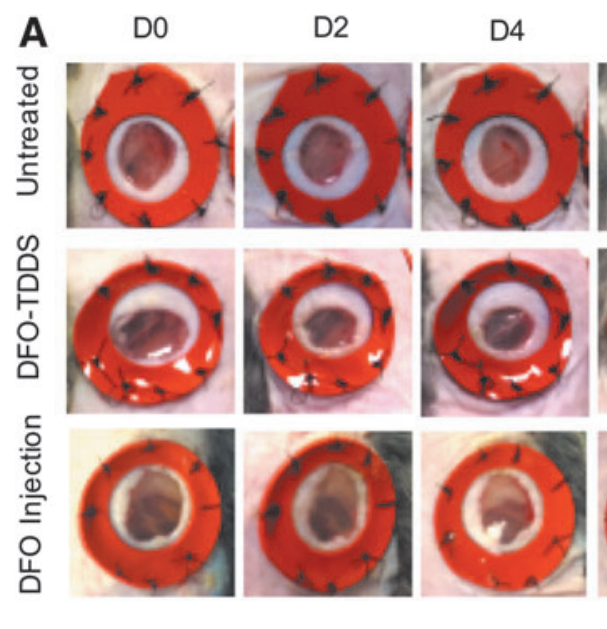

B

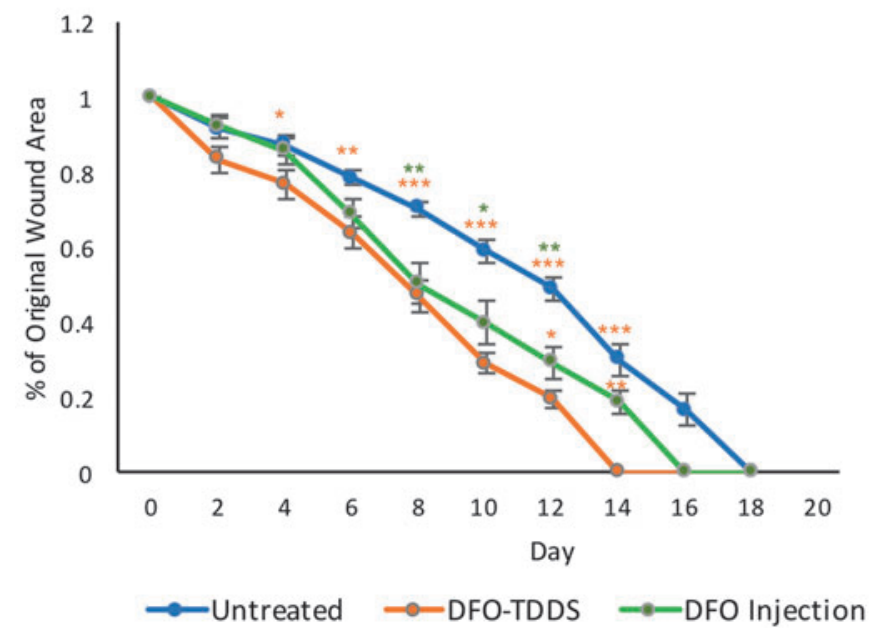

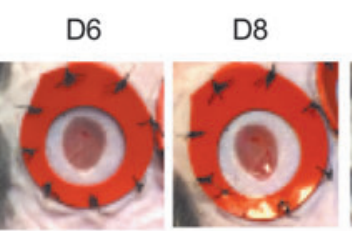
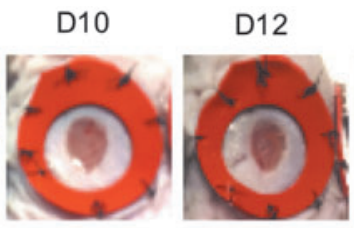

D14
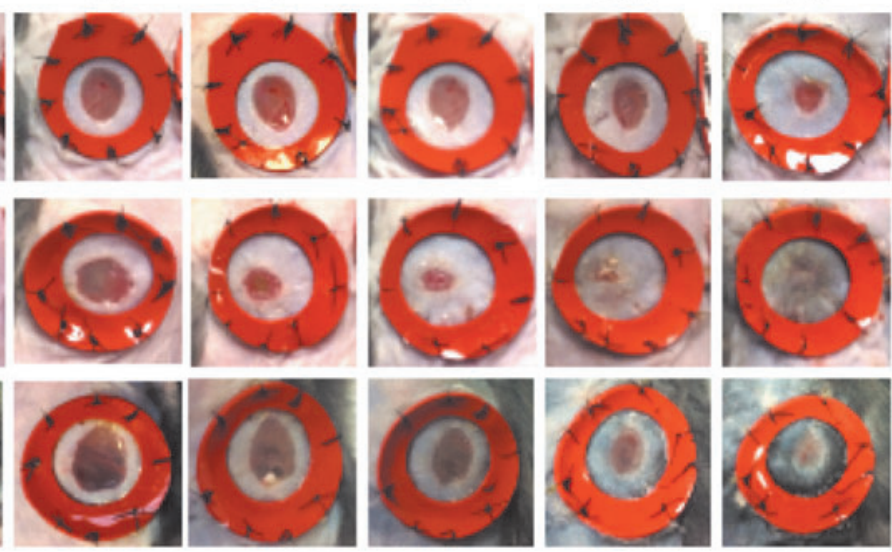

C
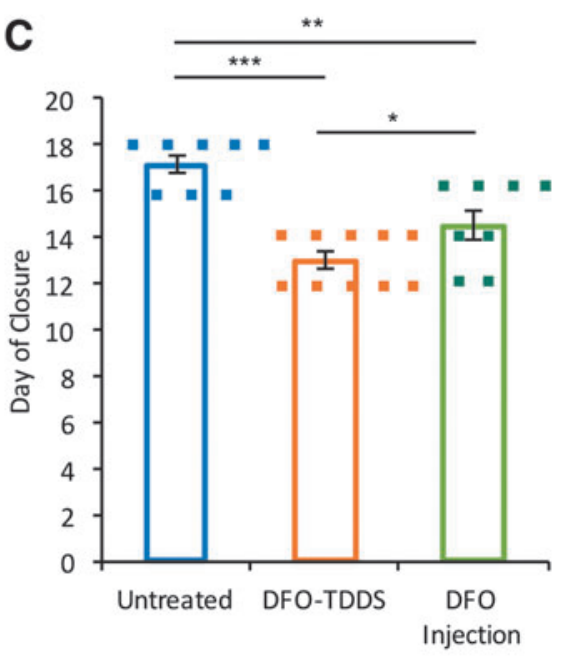

Figure 2. DFO-TDDS accelerates wound healing and is more effective than injected DFO at treating wounds in $H b S S$-BERK mice. (A) Photographs of wounds taken every other day after wounding until closure in the untreated, DFO-TDDS, and DFO injection groups. (B) Wound healing curves demonstrate reduction in wound size every other day until closure. (C) Denotes the time of closure of wounds. At least eight wounds were analyzed in each group. ${ }^{*} p<0.05,{ }^{* *} p<0.01$, ${ }^{* * *} p<0.001$ by ANOVA. DFO, deferoxamine; DFO-TDDS, deferoxamine transdermal delivery system.

treatment group demonstrated a well remodeled wound without excessive cell proliferation, uniformly bundled extracellular matrix, and the return of skin appendages. DFO-TDDS-treated wounds also displayed a thick dermis (more than $500 \mu \mathrm{m}$ thick), which was not observed in the untreated mice. Interestingly, the DFO injection group showed regions of active cell proliferation and the presence of disorganized extracellular matrix, indicating the wound had not resolved healing in this treatment group. Thus, sustained release of DFO through the TDDS is more effective in healing wounds in sickle cell mice.

Next, to understand why there was reduced Prussian blue stain in the DFO treatment and DFO injection groups, we aimed to understand the chemical reaction by which DFO binds iron. Iron is stable when bound to six oxygen atoms. Several chelators can provide these oxygen atoms, but DFO (Fig. 4C) is known to form the strongest bonds with iron, since it is a hexadentate ligand, sharing six oxygen atoms with $\mathrm{Fe}^{3+}$ to form ferrioxamine (Fig. 4D). Ferrioxamine is difficult to dissociate, even in dilute solutions $\left(10^{-5} \mathrm{M}\right)$ and thus withholds ferric iron from being available for the Perl's reaction. Thus, our results indicate that in the presence of DFO-TDDS, free iron in sickle cell wounds is chelated and no longer available to generate ROS and cellular damage.

\section{DISCUSSION}

Hemoglobin $\mathrm{S}$ is formed by a substitution of valine for glutamic acid (GAG $\rightarrow$ GTG) at position 6 in the $\beta$-globin chain of hemoglobin $\mathrm{A}^{4}$ The inheritance of two copies of this mutation $(H b S S)$, one 


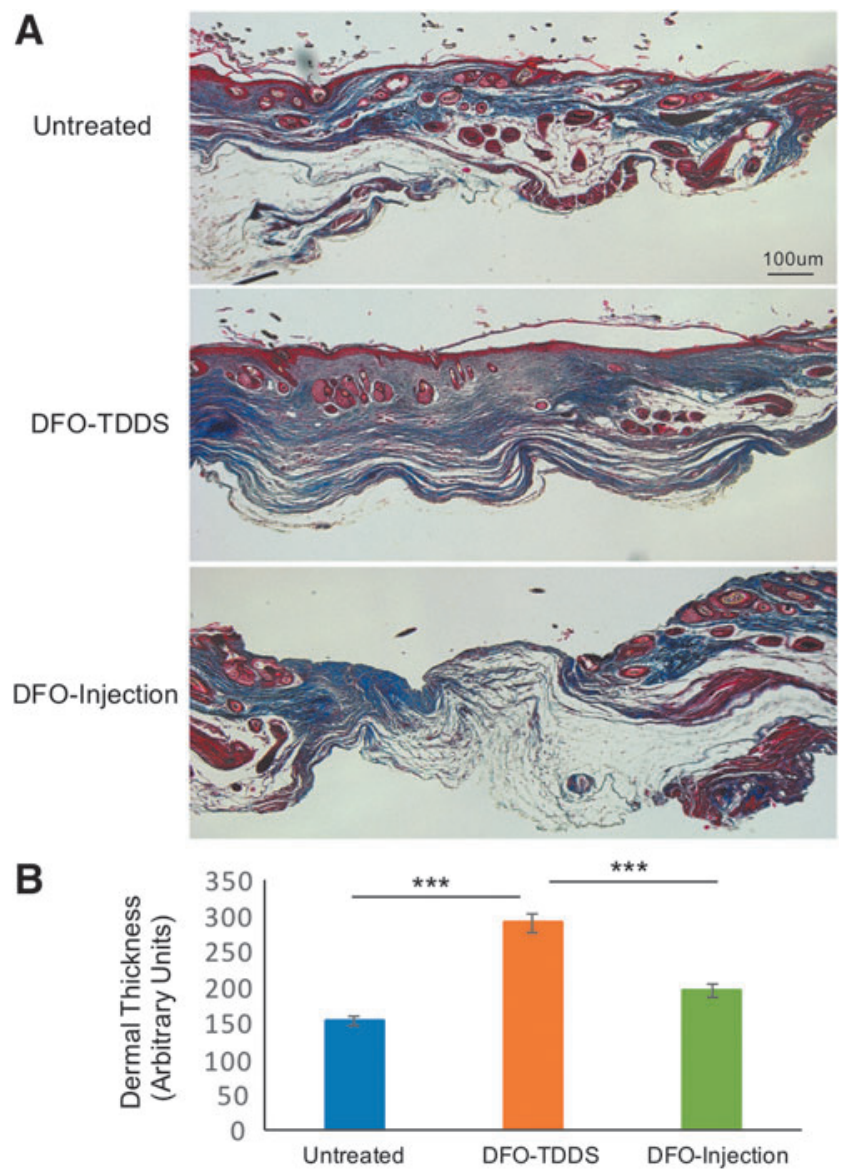

Figure 3. Wounds in HbSS-BERK mice treated with DFO-TDDS display increased collagen deposition and dermal thickness. (A) Trichrome staining of histological sections of healed wounds from $\mathrm{HbSS}$-BERK mice in the untreated, DFO-TDDS, and DFO injection groups demonstrates collagen deposition (blue). (B) Dermal thickness is measured from the photomicrographs of each wound in the DFO-TDDS-treated, DFO injection-treated, and untreated groups. ${ }^{* *} p<0.001$ by Student's $t$-test.

from each parent leads to homozygous SCD. HbSS polymerizes on deoxygenation, forming rigid sickleshaped erythrocytes. These erythrocytes impair blood flow and readily lyse leading to an accumulation of excessive free iron in the plasma and in tissues. ${ }^{4}$ The complications of SCD are myriad, but the most common acute events during childhood are pain, stroke, acute chest syndrome, and splenic sequestration. $^{29,30}$ As the patient advances into adulthood, progressive vasculopathy and hemochromatosis lead to end-organ damage, including chronic renal failure, stroke, avascular necrosis of bone, and pulmonary hypertension. ${ }^{31}$ Patients with SCD often develop SCUs, affecting physical function and the quality of life of the patient. ${ }^{24,32}$

DFO is a highly effective and nontoxic iron chelator that has been routinely used to remove excessive iron from patients with hemochromatosis. ${ }^{33,34}$ Due to its short half-life, it has been administered by subcutaneous or intravenous infusion, usually over 8-12 $\mathrm{h}$ and for 5-7 days/week. ${ }^{17,35}$ In this study, we hypothesized that local delivery of DFO to the wound site would improve wound healing in mice with SCD by chelating excessive free iron in the wound. However, the DFO molecule is hydrophilic and relatively large, making cellular diffusion difficult. ${ }^{19}$

To enable local delivery to both wounds and intact skin, we used a novel DFO-TDDS that contains DFO encapsulated in reverse micelles. ${ }^{23}$ When applied topically, it allows for DFO to easily diffuse through the impermeable stratum corneum and hydrophobic membranes, with targeted delivery into the dermis. ${ }^{23}$ We have previously demonstrated that DFO-TDDS improves wound healing in mice by enhancing new blood vessel formation and reducing production of $\mathrm{ROS}^{23}$

In this study, we were specifically interested in determining if DFO-TDDS could chelate iron in the skin of sickle cell mice and enhance wound healing. $H b S S$-BERK sickle cell mice were used for experiments. These mice contain $>99 \%$ human sickle hemoglobin $^{25}$ and display similar tissue impairments as evidenced in patients with SCD, including hemeinduced vasoocclusion, ${ }^{36}$ decreased epidermal and dermal thickness, ${ }^{27}$ hyperalgesia, ${ }^{27}$ and mechanical allodynia. ${ }^{37}$ In our experiments, $H b S S$-BERK mice demonstrated slower wound healing compared with wild-type mice. Healed wounds in untreated $\mathrm{HbSS}$ BERK mice displayed increased iron in the dermis by Perl's Prussian blue stain. Regions of high iron accumulation directly correlated with reduced dermal thickness (Fig. 5).

Daily application of DFO-TDDS on wounds significantly accelerated wound closure, increased dermal thickness, and resulted in deposition of organized collagen bundles compared with both untreated wounds and wounds injected with DFO solution daily. All mice were given buprenorphine slow-release as an analgesic during wounding to counter pain, which is a characteristic feature of the HbSS-BERK mice. ${ }^{26,27,38}$ DFO-TDDS-treated wounds displayed negligible iron in the dermis (Fig. 5), indicating iron chelation by the formation of ferrioxamine. While the DFO injection-treated wounds also displayed negligible iron in the dermis, the wounds in this treatment group were still in the proliferative state with disorganized extracellular matrix, indicating that a single dose of DFO given once a day is not as effective as sustained release of DFO throughout the wound healing process.

Ferric iron $\left(\mathrm{Fe}^{3+}\right)$ is much more stable under aerobic conditions in relation to ferrous iron $\left(\mathrm{Fe}^{2+}\right)$ and is of greater significance when assessing the value of different chelators. ${ }^{18}$ The positive charge 
A
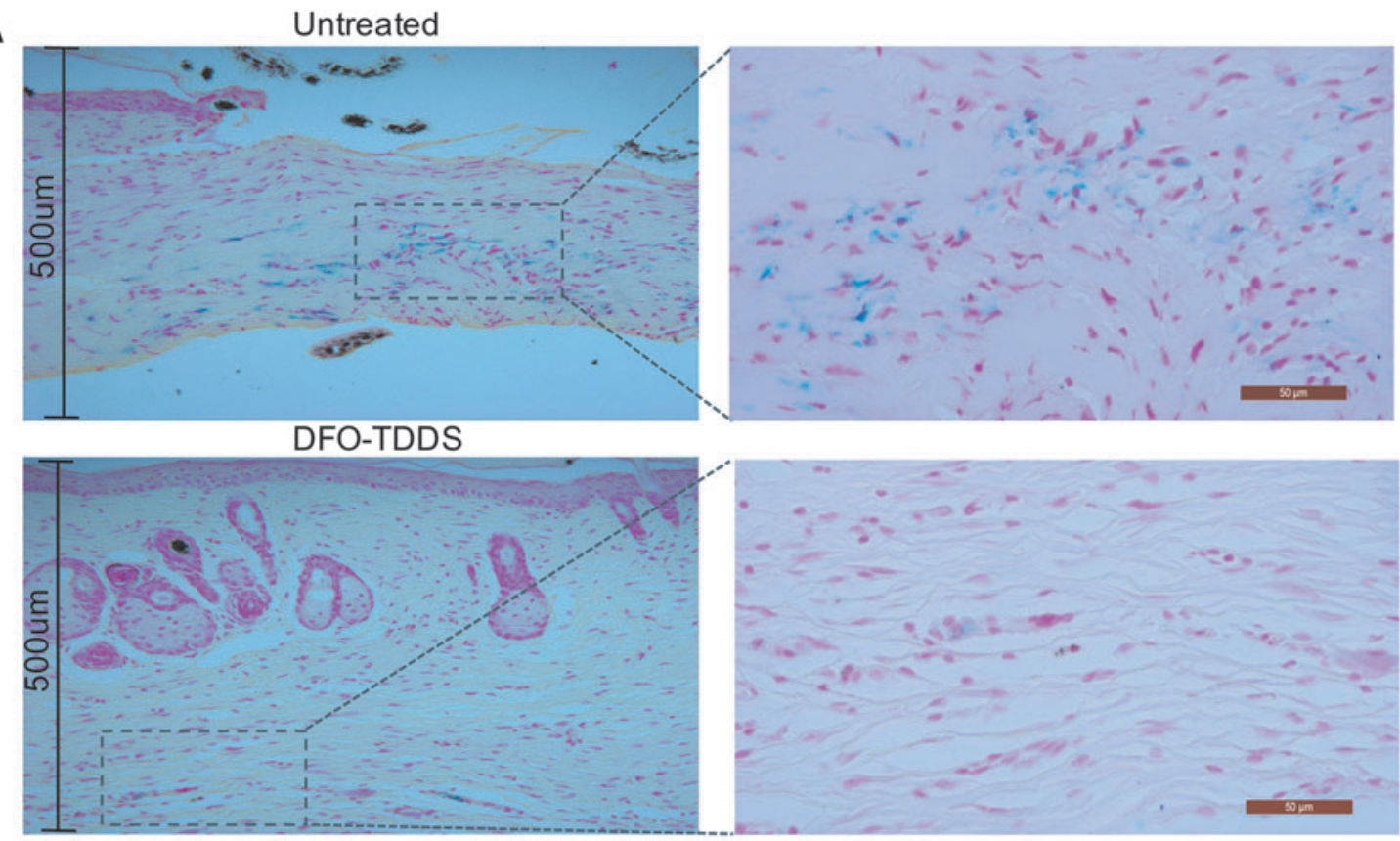

DFO-Injection

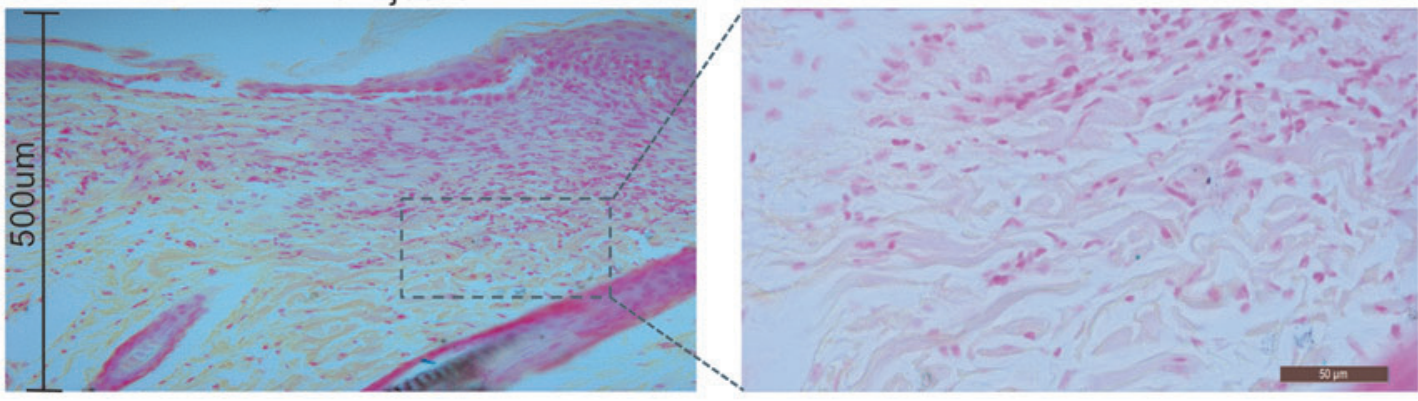

B

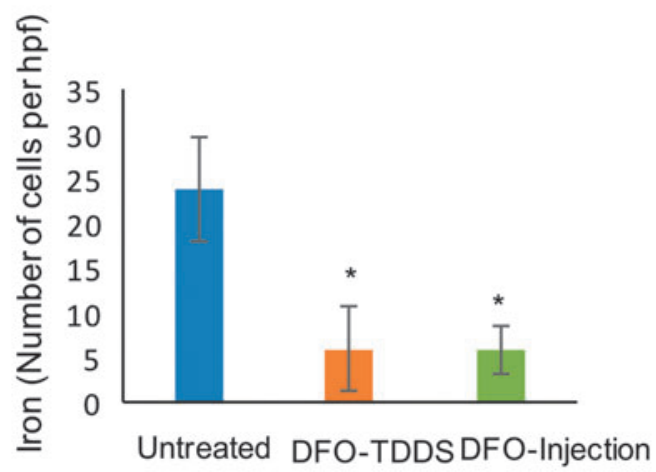

C<smiles>CC(=O)ON(O)CCCCCNC(=O)CCC(=O)N(O)CCCCCNC(=O)CCC(=O)N(O)CCCCN</smiles>

D

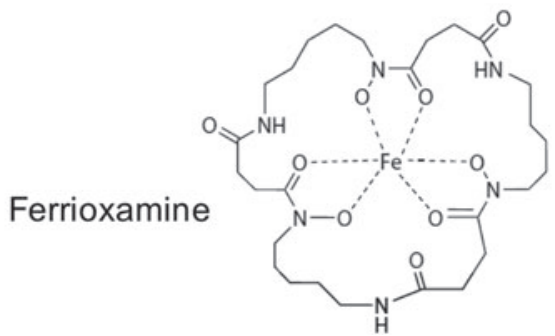

Figure 4. Wounds in HbSS-BERK mice treated with DFO-TDDS display reduced iron by Perl's Prussian blue stain. (A, B) Healed wounds in the untreated, DFO-TDDS-treated, and DFO injection-treated groups were subjected to Perl's Prussian blue stain. Iron (blue) was detected in the untreated wounds but not in DFO-TDDS- or DFO injection-treated groups, indicating chelation of iron by DFO. (C) Chemical structure of deferoxamine. (D) Chemical structure of ferrioxamine, the stable chemical compound formed by binding of iron $\left(\mathrm{Fe}^{3+}\right)$ and deferoxamine. ${ }^{*} p<0.05$ by Student's $t$-test. 


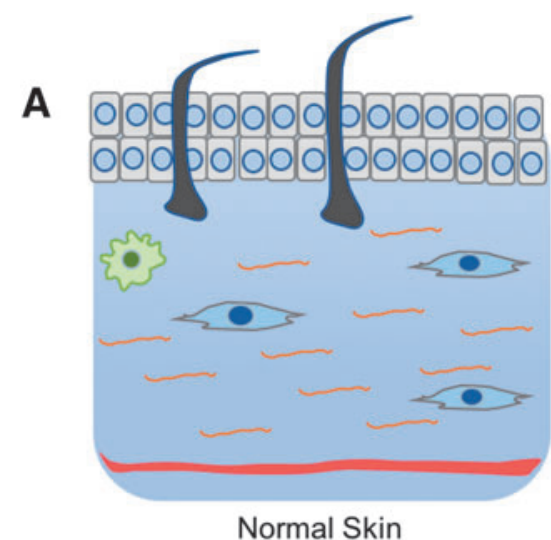

Normal Skin

$\mathbf{F}$

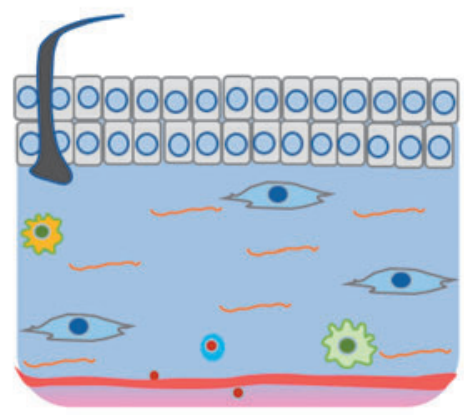

Healed Sickle Cell Ulcer

$\mathbf{E}$

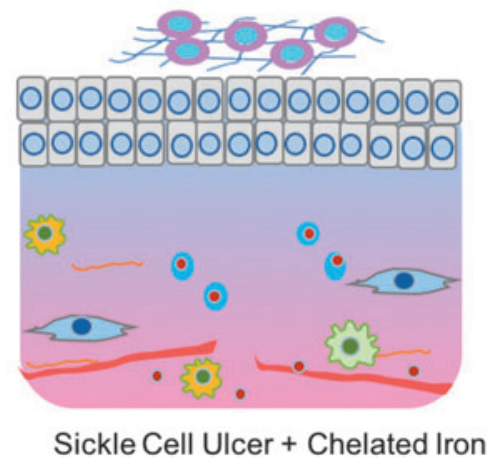

B

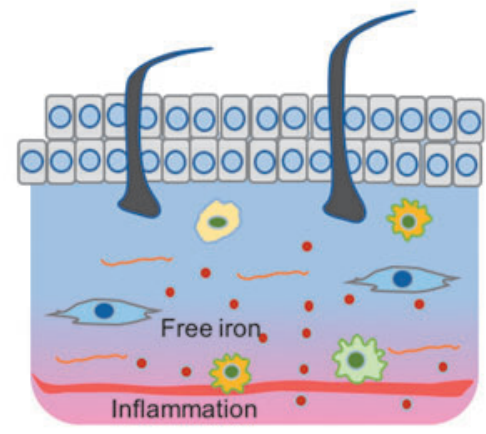

Sickle Cell Skin

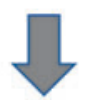

C

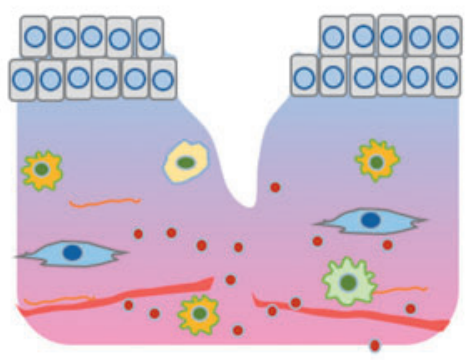

Sickle Cell Ulcer

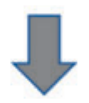

D

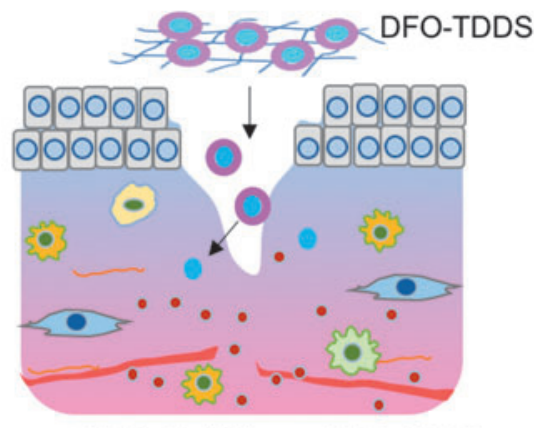

Sickle Cell Ulcer + DFO-TDDS

Figure 5. Schematic demonstration of the effectiveness of DFO-TTDS in healing sickle cell ulcers. (A) Normal skin is unlike (B) skin in sickle cell disease, which has excessive free iron, increased inflammation, and lesser dermal strength. Thus, sickle cell skin is exposed to (C) recurrent breakage in regions of excessive iron accumulation causing sickle cell ulcers. (D) Topical application of DFO-TDDS on sickle cell ulcers results in the release of DF0 into the dermis and $(E)$ chelation of excessive free iron in a 1:1 ratio. (F) Continued application of DFO-TDDS until closure of the ulcer results in a thicker dermis and a remodeled wound, which can resume normal homeostasis.

of ferric iron creates a particularly high charge density within the atom, thereby predisposing it to forming bonds with other atoms that possess high charge densities. ${ }^{18}$ Chelators such as DFO have regions of polarization that serve as strong binding points for highly charged ferric cations. As DFO has six binding sites with ferric iron, it is labeled a hexadentate ligand. ${ }^{18}$ Hexadentate ligands exhibit greater binding strength at lower concentrations than others, such as bidentate ligands, and thus are less likely to dissociate and form hydroxyl radicals. ${ }^{18}$ These chemical properties make DFO a highly efficient drug to chelate excessive ferric iron in the skin. Once chelated, ferrioxamine most likely 
is excreted via exfoliation of epidermal cells, through sweat, or might enter the blood stream and is excreted via the kidney. ${ }^{39,40}$

Our results indicate for the first time that removal of iron from the dermis of $H b S S$ sickle cell mice directly correlates with improvement of wound healing. Reducing iron locally in the skin using an FDA-approved chelator, DFO significantly enhances time to wound closure, dermal thickness, and wound remodeling. Sustained delivery of DFO through the DFO-TDDS is more effective in closing wounds compared with a single bolus of DFO injected locally. DFO-TDDS is currently being manufactured, sterilized, and packaged under good manufacturing practice in an FDA-compliant facility. This will allow for rapid translation for treatment of patients with SCUs.

\section{INNOVATION}

SCUs are a significant health care burden. There is no effective therapy for their prevention or treatment. Beyond standard debridement and ensuring that the wound remains clean, few solutions exist. To address SCUs, we have developed DFOTDDS, which enables DFO to be released into both the intact skin and wounds. In this study, we establish the efficacy of DFO-TDDS for treating SCUs where no alternative strategy currently exists. The TDDS also introduces a technology through which other small-molecule drugs can be delivered into the skin for treating wounds in other impaired states. DFO-TDDS has been scaled for human application in an FDA-compliant facility.

\section{ACKNOWLEDGMENTS AND FUNDING SOURCES}

We thank Joe Rimsa and his staff at TauTona Group for manufacture and delivery of the DFO-
TDDS, Yujin Park for her assistance in tissue processing, Ritu Jha for breeding and phenotyping mice, and Theresa Carlomagno and Barb Benson for administrative support.

\section{ABOUT THE AUTHORS}

Geoffrey C. Gurtner, MD, FACS, and Michael T. Longaker, MD, MBA, FACS, are Professors in the Department of Surgery at Stanford and Co-Directors of the Hagey Laboratory for Pediatric Plastic Surgery Research where their laboratories study the mechanisms underlying impaired wound healing. Caterina P. Minniti, MD, is Professor of Clinical Medicine and Pediatrics at Albert Einstein College of Medicine and Director of the Sickle Cell Center for adults at Montefiore Medical Center. Kalpna Gupta, PhD, is Professor of Medicine at the University of Minnesota where she studies pain and vascular diseases in sickle cell mice. Clark A. Bonham, BS, is a research fellow in Dr. Gurtner's Laboratory. Melanie Rodrigues, $\mathbf{P h D}$, is an Instructor in the Department of Surgery at Stanford where she conducts research in wound healing.

\section{AUTHOR DISCLOSURE AND GHOSTWRITING}

G.C.G. and M.T.L. have filed patents on this work. The article was written by the authors and ghostwriting services were not used.

\section{REFERENCES}

1. Penne JR, Goodman BM, Chen IA. Sickle cell disease \& wound care: Lower extremity ulcers in "crisis." Todays Wound Clinic 2015;9:25-27.

2. Delaney KM, Axelrod KC, Buscetta A, et al. Leg ulcers in sickle cell disease: Current patterns and practices. Hemoglobin 2013;37:325-332.

3. Rees DC, Williams TN, Gladwin MT. Sickle-cell disease. Lancet 2010;376:2018-2031.
4. Bunn HF. Pathogenesis and treatment of sickle cell disease. N Engl J Med 1997;337: 762-769.

5. Hebbel RP, Boogaerts MA, Eaton JW, Steinberg $\mathrm{MH}$. Erythrocyte adherence to endothelium in sickle-cell anemia. A possible determinant of disease severity. N Engl J Med 1980;302:992995.
6. Hebbel RP, Morgan WT, Eaton JW, Hedlund BE. Accelerated autoxidation and heme loss due to instability of sickle hemoglobin. Proc Natl Acad Sci U S A 1988;85:237-241.

7. Van Avondt K, Schimmel M, Bulder I, et al. Free iron in sera of patients with sickle cell disease contributes to the release of neutrophil extracellular traps. Blood 2016;128:16. 
8. Chirico EN, Pialoux V. Role of oxidative stress in the pathogenesis of sickle cell disease. IUBMB Life 2012;64:72-80.

9. Reiter CD, Wang X, Tanus-Santos JE, et al. Cell-free hemoglobin limits nitric oxide bioavailability in sickle-cell disease. Nat Med 2002;8:1383-1389.

10. Walter PB, Fung EB, Killilea DW, et al. Oxidative stress and inflammation in iron-overloaded patients with beta-thalassaemia or sickle cell disease. Br J Haematol 2006;135:254-263.

11. Minniti CP, Delaney KM, Gorbach AM, et al. Vasculopathy, inflammation, and blood flow in leg ulcers of patients with sickle cell anemia. Am J Hematol 2014;89:1-6.

12. Minniti CP, Eckman J, Sebastiani P, Steinberg $\mathrm{MH}$, Ballas SK. Leg ulcers in sickle cell disease. Am J Hematol 2010;85:831-833.

13. Ladizinski B, Bazakas A, Mistry N, Alavi A, Sibbald RG, Salcido R. Sickle cell disease and leg ulcers. Adv Skin Wound Care 2012;25:420-428.

14. Ballas SK. Treatment of painful sickle cell leg ulcers with topical opioids. Blood 2002;99:1096.

15. Franchini M, Gandini G, de Gironcoli M, Vassanelli A, Borgna-Pignatti C, Aprili G. Safety and efficacy of subcutaneous bolus injection of deferoxamine in adult patients with iron overload. Blood 2000; 95:2776-2779.

16. Inati A, Khoriaty E, Musallam KM, Taher AT. Iron chelation therapy for patients with sickle cell disease and iron overload. Am J Hematol 2010;85: 782-786.

17. Vichinsky E, Onyekwere 0, Porter J, et al. A randomised comparison of deferasirox versus deferoxamine for the treatment of transfusional iron overload in sickle cell disease. $\mathrm{Br} \mathrm{J}$ Haematol 2007:136:501-508.

18. Porter JB, Huehns ER, Hider RC. The development of iron chelating drugs. Baillieres Clin Haematol 1989;2:257-292.

19. Porter JB. Deferoxamine pharmacokinetics. Semin Hematol 2001;38:63-68.

20. Kalpatthi R, Peters B, Kane I, et al. Safety and efficacy of high dose intravenous desferriox- amine for reduction of iron overload in sickle cell disease. Pediatr Blood Cancer 2010;55:13381342.

21. Thangarajah $H$, Yao D, Chang El, et al. The molecular basis for impaired hypoxia-induced VEGF expression in diabetic tissues. Proc Natl Acad Sci U S A 2009;106:13505-13510.

22. Duscher $D$, Januszyk $M$, Maan $Z N$, et al. Comparison of the hydroxylase inhibitor dimethyloxalylglycine and the iron chelator deferoxamine in diabetic and aged wound healing. Plast Reconstr Surg 2017;139:695e-706e.

23. Duscher D, Neofytou E, Wong VW, et al. Transdermal deferoxamine prevents pressure-induced diabetic ulcers. Proc Natl Acad Sci U S A 2015; 112:94-99

24. Minniti CP, Kato GJ. Critical Reviews: How we treat sickle cell patients with leg ulcers. Am J Hematol 2016;91:22-30.

25. Paszty C, Brion CM, Manci E, et al. Transgenic knockout mice with exclusively human sickle hemoglobin and sickle cell disease. Science 1997; 278:876-878.

26. Cain DM, Vang D, Simone DA, Hebbel RP, Gupta $\mathrm{K}$. Mouse models for studying pain in sickle disease: Effects of strain, age, and acuteness. $\mathrm{Br} \mathrm{J}$ Haematol 2012;156:535-544.

27. Kohli DR, Li Y, Khasabov SG, et al. Pain-related behaviors and neurochemical alterations in mice expressing sickle hemoglobin: Modulation by cannabinoids. Blood 2010;116:456-465.

28. Galiano RD, Michaels J 5th, Dobryansky M, Levine JP, Gurtner GC. Quantitative and reproducible murine model of excisional wound healing. Wound Repair Regen 2004:12:485-492.

29. Platt OS. The acute chest syndrome of sickle cell disease. N Engl J Med 2000;342:1904-1907.

30. Platt OS, Brambilla DJ, Rosse WF, et al. Mortality in sickle cell disease. Life expectancy and risk factors for early death. N Engl J Med 1994;330:1639-1644.

31. Gladwin MT, Vichinsky E. Pulmonary complications of sickle cell disease. N Engl J Med 2008; 359:2254-2265.
32. Umeh NI, Ajegba B, Buscetta AJ, Abdallah KE, Minniti CP, Bonham VL. The psychosocial impact of leg ulcers in patients with sickle cell disease: I don't want them to know my little secret. PLoS One 2017;12:e0186270.

33. Silliman CC, Peterson VM, Mellman DL, Dixon DJ, Hambidge KM, Lane PA. Iron chelation by deferoxamine in sickle cell patients with severe transfusion-induced hemosiderosis: A randomized, double-blind study of the dose-response relationship. J Lab Clin Med 1993;122:48-54.

34. Brittenham GM, Griffith PM, Nienhuis AW, et al. Efficacy of deferoxamine in preventing complications of iron overload in patients with thalassemia major. N Engl J Med 1994;331:567-573.

35. Porter JB. Practical management of iron overload. $\mathrm{Br} J$ Haematol 2001;115:239-252.

36. Belcher JD, Chen C, Nguyen J, et al. Heme triggers TLR4 signaling leading to endothelial cell activation and vaso-occlusion in murine sickle cell disease. Blood 2014;123:377-390.

37. Garrison SR, Kramer AA, Gerges NZ, Hillery CA Stucky CL. Sickle cell mice exhibit mechanical allodynia and enhanced responsiveness in light touch cutaneous mechanoreceptors. Mol Pain 2012;8:62.

38. Tran H, Gupta M, Gupta K. Targeting novel mechanisms of pain in sickle cell disease. Blood 2017:130:2377-2385.

39. Weintraub LR, Demis DJ, Conrad ME, Crosby WH. Iron excretion by the skin. Selective localization of iron-59 in epithelial cells. Am J Pathol 1965;46:121-127.

40. Adams BD, Lazova R, Andrews NC, Milstone LM. Iron in skin of mice with three etiologies of systemic iron overload. J Invest Dermatol 2005;125:1200-1205.

\section{Abbreviations and Acronyms}

DFO = deferoxamine

DFO-TDDS $=$ deferoxamine transderma delivery system

$\mathrm{ROS}=$ reactive oxygen species

SCD $=$ sickle cell disease

SCU $=$ sickle cell ulce 